\title{
Interaction of Irbesartan with Bovine Hemoglobin Using Spectroscopic Techniques and Molecular Docking
}

\author{
Ai-Ping Yang, ${ }^{1,2}$ Mei-Hua Ma, ${ }^{1,2}$ Xiao-Hua Li, ${ }^{1,2}$ and Mao-Yun Xue ${ }^{1,2}$ \\ ${ }^{1}$ Department of Engineering Technology, Jiangsu Institute of Economic and Trade Technology, \\ Guanghua Road 104, Nanjing City 210007, Jiangsu Province, China \\ ${ }^{2}$ Research and Development Centre of Food Safety Engineering Technique, Guanghua Road 104, \\ Nanjing City 210007, Jiangsu Province, China
}

Correspondence should be addressed to Xiao-Hua Li, xiaohuali57@163.com

Copyright (C) 2012 Ai-Ping Yang et al. This is an open access article distributed under the Creative Commons Attribution License, which permits unrestricted use, distribution, and reproduction in any medium, provided the original work is properly cited.

\begin{abstract}
The binding of irbesartan to bovine hemoglobin $(\mathrm{BHb})$ has been investigated for the first time by using UV-Vis absorption, fluorescence, circular dichroism (CD), and molecular docking. The binding site number $n$ and binding constant $K$ were calculated to be 1 and $2.41 \times 10^{5} \mathrm{M}^{-1}$, respectively. The alternations of protein secondary structure in the presence of irbesartan was demonstrated using CD spectroscopy. Furthermore, molecular docking indicated that irbesartan could bind to the site 2 of $\mathrm{BHb}$. The analysis of the binding site of irbesartan within the BHb molecule suggested that hydrophobic interaction, hydrogen bond formation, and electrostatic interaction could account for the binding of irbesartan. The hydrogen bond of irbesartan with His87 in the $\mathrm{C}$ chain of $\mathrm{BHb}$ has been formed. The electrostatic energy, van der Waals energy, and binding free energy were calculated to be $-460.3,-224.2$, and $-684.5 \mathrm{kcal} \cdot \mathrm{moL}^{-1}$, respectively.
\end{abstract}

Keywords: Interaction, irbesartan, bovine hemoglobin, spectroscopic technique, molecular docking

\section{Introduction}

A full understanding of the modes of drugs' action requires the study on the interactions of all possible chemical and biological targets, including amino acids, hormones, peptides, and proteins. Studies based on drugs-protein interactions improve the armory of molecular tools for detecting and manipulating the biological roles of biomacromolecules. Such studies also crucially determine the bioavailability and toxicology of some injected drug. Hemoglobin $(\mathrm{Hb})$ is an important functional protein for reversible oxygen carrying and storage in animals. It also transports carbon dioxide to regulate the $\mathrm{pH}$ of blood. $\mathrm{Hb}$ contains four globin chains, the two of them are $\alpha$-chains and the others are $\beta$-chains [1]. There are four oxygen-binding sites on the $\mathrm{Hb}$ molecule. Generally speaking, when the drugs get into the corresponding target, they will be stored and transported through the blood plasma firstly. Inevitably, they have the interaction with $\mathrm{Hb}$. Therefore, the study on Hb-drug interaction may provide some important theoretic information of the structural features that determine the therapeutic effectiveness 


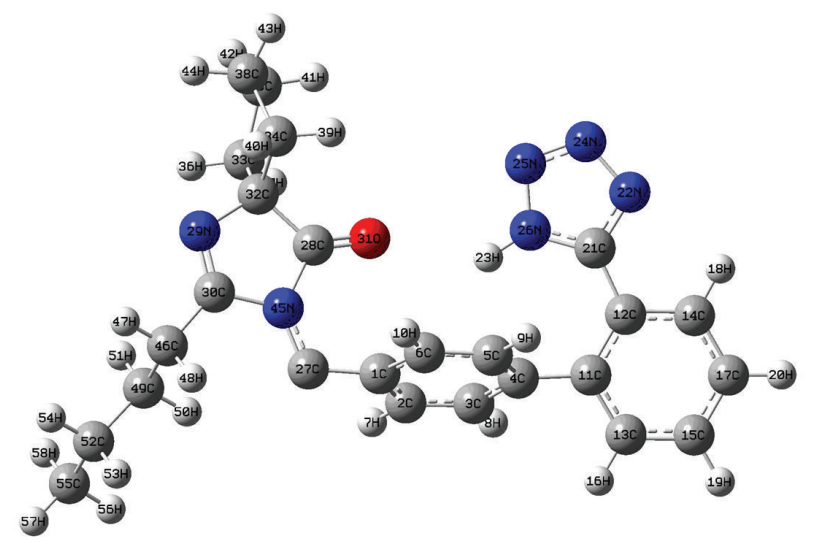

Figure 1: The chemical structure of irbesartan.

of drugs [2] and become an important research filed in life science, chemistry, biologic technology, medicine, and molecular functional design. Nowadays, many researches on the binding of drugs to proteins have been carried out [3], but little report on the interaction between protein and the angiotensin II drugs.

Irbesartan (Figure 1) is one of the new angiotensin II drugs. It is a potent and selective angiotensin II subtype receptor antagonist indicated for the use in patients with type 2 diabetes and nephropathy. And it is effective in reducing hypertension. Hypertension is the most prevalent modifiable risk factor for cardiovascular disease. Epidemiologically, the lower the blood pressure, the better the cardiovascular prognosis. The major endocrine factor to control blood pressure is angiotensin. The octapeptide angiotensin II is a potent vasoconstrictor. The predominant effects of angiotensin II, that is, its action on blood pressure and water balance, are mediated by angiotensin $\mathrm{AT}_{1}$ receptors [4].

Study on the interaction of drugs to proteins can elucidate the properties of drug-protein complex. For a better understanding, the study of interaction between irbesartan and $\mathrm{Hb}$ is much needed. The mode of interaction, binding constant, and binding sites are significant in our research. To characterize the interaction at the molecular level, optical techniques are great tools because of their high sensitivity, rapidity, and ease of implementation. The flexible protein-ligand docking plays an increasingly important role in the identification of potential lead compounds in the drug discovery process. It can dock flexible ligands into an ensemble of protein structures which represents the flexibility, point mutations, or alternative models of a protein [5]. In a virtual screening experiment, each molecule is docked and finally the molecules in the database are reranked according to the score.

In this paper, the fluorescence quenching technique and UV-Vis absorption spectra were applied to study the interactions of irbesartan and Bovine hemoglobin (BHb). Furthermore, far-ultraviolet circular dichroism (CD) spectroscopy was used for demonstrating the changes of protein secondary structure. Finally, in order to obtain a clearer insight into the residues involved in the interaction, the irbesartan binding mode to $\mathrm{BHb}$ was studied using automated flexible docking approach. Experimental observations and theoretical data could be helpful to understand transports of irbesartan in the blood. 


\section{Experimental}

\subsection{Materials}

Bovine hemoglobin $(\mathrm{BHb})$ was purchased from Sigma Chemical Company and used without further purification. Irbesartan was obtained from Shanghai Institute of Materia Medica Chinese Academy of Sciences. BHb solutions $\left(1 \times 10^{-5} \mathrm{M}\right.$ and $\left.5 \times 10^{-6} \mathrm{M}\right)$ were prepared in $0.05 \mathrm{M}$ Tris-HCl buffer solution with $\mathrm{PH} 7.4$ containing $0.1 \mathrm{M} \mathrm{NaCl}$ to maintain the ionic strength of the solution. The irbesartan solution $\left(1 \times 10^{-4} \mathrm{M}\right)$ was prepared by dissolving irbesartan in absolute methanol solution. Absolute methanol was purchased from TEDIA Company in America. All other reagents and solvents were of analytical reagent grade. All aqueous solutions were prepared using newly double distilled water.

\subsection{Apparatus and Methods}

UV-Vis spectra of samples were recorded on a Cary 50 spectrophotometer equipped with $1.0 \mathrm{~cm}$ quartz cell at $298 \mathrm{~K}$, using Cary Winuv software.

A Cary Eclipse spectrofluorimeter (Varian, Australia) equipped with $1.0 \mathrm{~cm}$ quartz cells was utilized to measure the fluorescence emission spectra, using $5.0 \mathrm{~nm} \times 10.0 \mathrm{~nm}$ slit widths. Different ratios of irbesartan and $\mathrm{BHb}$ solutions were prepared before the experiments. They were $0,0.1,0.2,0.4$, 0.8 , and 1.2 , respectively. The emission spectra were recorded in the range of 300-400 nm using the excitation wavelength of $280 \mathrm{~nm}$.

The CD spectrums were obtained on a Jasco J-810 spectropolarimeter (Jasco Co., Ltd., Tokyo, Japan). The samples were transferred to a quartz cell with a $1 \mathrm{~cm}$ light-path length. Molecular ellipticities were measured in the range of $200-225 \mathrm{~nm}$. The optical chamber of the CD spectrometer was deoxygenated with dry nitrogen before using and kept in nitrogen atmosphere throughout the experiment.

The crystal structure of $\mathrm{BHb}$ used for molecular docking was obtained from the structure having Protein Data Bank (PDB) identifier 1G09. In order to optimize hydrogen positions, remove interatomic bumps, and correct the covalent geometry, the structure of BHb was energy-minimized with the CHARMm force filed. Waters and all other HETATM molecules were removed from the BHb PDB file. Polar hydrogen atoms and Gasteiger charges were added to prepare the BHb molecule for docking. Protein-ligand docking was carried out with the flexible docking tool in the Discovery Studio 2.1 (DS 2.1) software of Accelrys company in America. The flexible of the residues which surround the active site of $\mathrm{BHb}$ and the ligand were all considered in the docking procedure. In this work, the selection of flexible residues for the induced fit is based on the active site of the $\mathrm{BHb}$.

\section{Results and Discussion}

\subsection{Analysis of $U V$-Visible Spectroscopy}

UV-Vis absorption measurement is a very simple and applicable method to explore the structural change which may be caused by the complex formation. The UV-Vis absorption spectra of BHb with and without irbesartan were showed in Figure 2. BHb has four heme groups located in the crevices near 


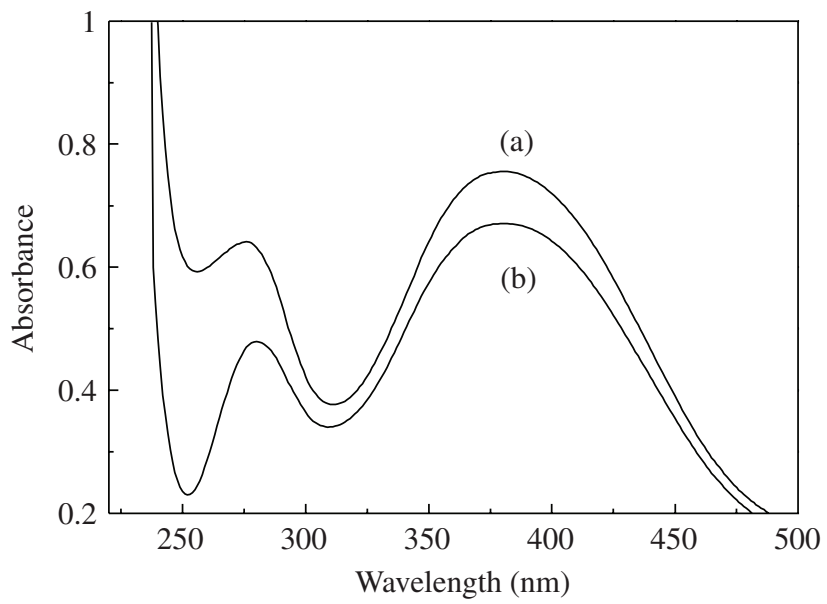

Figure 2: UV-Vis absorption spectrum of (a) $\mathrm{BHb}$ and (b) irbesartan. $\mathrm{BHb}=0.4$ system.

the surface of the molecule, and there were two peaks (275 and $380 \mathrm{~nm})$ in its absorption spectrum. The absorption peak $(275 \mathrm{~nm}$ ) is engendered by the phenylalanine (Phe), tyrosine (Tyr), and tryptophane (Trp) residues [6]. In this peak of irbesartan-BHb system showed a slight Einstein shift. The peak at $380 \mathrm{~nm}$ corresponds to the soret-band of $\mathrm{BHb}$. The absorption spectrum of soret-band in presence of irbesartan did not show any spectral change, which indicated that all four heme groups bound to hemoglobin were not directly attacked and degraded by irbesartan [6]. As can be seen in Figure 2, the main change in the spectra is the decline of the absorbance. These results indicate that the decline of the absorbance is due to the interaction between irbesartan and $\mathrm{BHb}$, the formation of the complex, and the changed microenvironment around $\mathrm{BHb}$.

\subsection{Analysis of Fluorescence Spectroscopy}

For macromolecules, the fluorescence measurements could provide some information of the binding of small molecule substance to protein on the molecular level, such as the binding mechanism and binding constants, In order to ascertain the value of the association constant and the binding sites in the interaction between irbesartan and $\mathrm{BHb}$, fluorescence spectroscopy is much needed. Fluorescence quenching is the decrease of the quantum yield of fluorescence from a fluorophore induced by a variety of molecular interactions with quencher molecule, such as excited-state reaction, molecules rearrange, energy transfer, ground state complex formation, and collision quenching. The fluorescence quenching behavior is known to occur mainly through a dynamic process or a static process. Collisional or dynamic quenching refers to a process that the fluorophore and the quencher come into contact during the lifetime of the excited state, whereas static quenching refers to the formation of the complex between quencher and fluorophore.

Figure 3 showed that the fluorescence emission spectra of $\mathrm{BHb}$ in the absence and presence of irbesartan. From Figure 3(a), it can be seen that BHb has a strong fluorescence emission peak at $334 \mathrm{~nm}$. The gradual addition of irbesartan and to $\mathrm{BHb}$ solution results in considerable quenching of $\mathrm{BHb}$ fluorescence intensity. The intrinsic fluorescence peak at $336 \mathrm{~nm}$ originates from the Trp and Tyr 


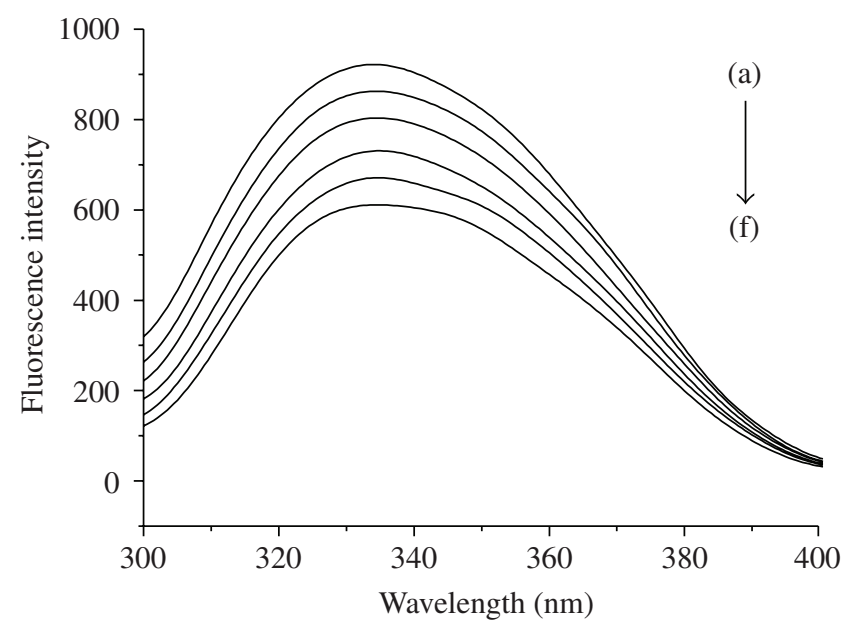

Figure 3: Emission fluorescence spectra of $\mathrm{BHb}$ with a concentration ratio of irbesartan and BHB: (a) 0 , (b) 0.1 , (c) 0.2 , (d) 0.4, (e) 0.8, and (f) 1.2 .

residues mainly. In order to confirm the quenching mechanism, the fluorescence dynamic quenching data were analyzed by the Stern-Volmer equation [7]:

$$
\frac{F_{0}}{F}=1+K_{\mathrm{sv}}[Q]=1+K_{q} \tau_{0}[Q]
$$

where $F_{0}$ and $F$ represent the relative fluorescence intensities in the absence and presence of quencher, respectively, and $[Q]$ is the concentration of the quencher. $K_{q}$ is the quenching rate constant of bimolecular, while $\tau_{0}$ is the average lifetime of the $\mathrm{BHb}$ without quencher. $K_{\mathrm{sv}}$ is the Stern-Volmer quenching constant.

Figure 4 displayed the Stern-Volmer plots of the quenching of BHb Trp and Tyr residues fluorescence caused by irbesartan. The plots showed that within the investigated concentrations, the results agreed with the Stern-Volmer equation. The average lifetime of the bimolecule without quencher is $10^{-8} \mathrm{~s}$, and the maximum scatter collision quenching constant of various quenchers with the biopolymer is $2.0 \times 10^{10} \mathrm{M}^{-1} \cdot \mathrm{s}^{-1}$ [7]. According to (3.1), the values of $K_{\mathrm{sv}}$ and $K_{q}$ were calculated to be $2.49 \times 10^{4} \mathrm{M}^{-1}$ and $2.49 \times 10^{12} \mathrm{M}^{-1} \cdot \mathrm{s}^{-1}$, respectively. Therefore, the static quenching could be the main mechanism of the quenching of $\mathrm{BHb}$ by irbesartan.

The fluorescence quenching came from the formation of complex between irbesartan and $\mathrm{BHb}$, then the equilibrium between free and bound molecule could be given by following equation [8]:

$$
\log \left[\frac{F_{0}-F}{F}\right]=\log K+n \log [Q]
$$

where in the present case, $K$ is the binding constant, reflecting the degree of irbesartan and $\mathrm{BHb}$, and $n$ is the number of binding sites per $\mathrm{BHb}$, specifying the number of irbesartan bound to a $\mathrm{BHb}$ macromolecule. Thus, plots of $\log \left[\left(F_{0}-F\right) / F\right]$ versus $\log [Q]$ gave a straight line (shown in Figure 5) 


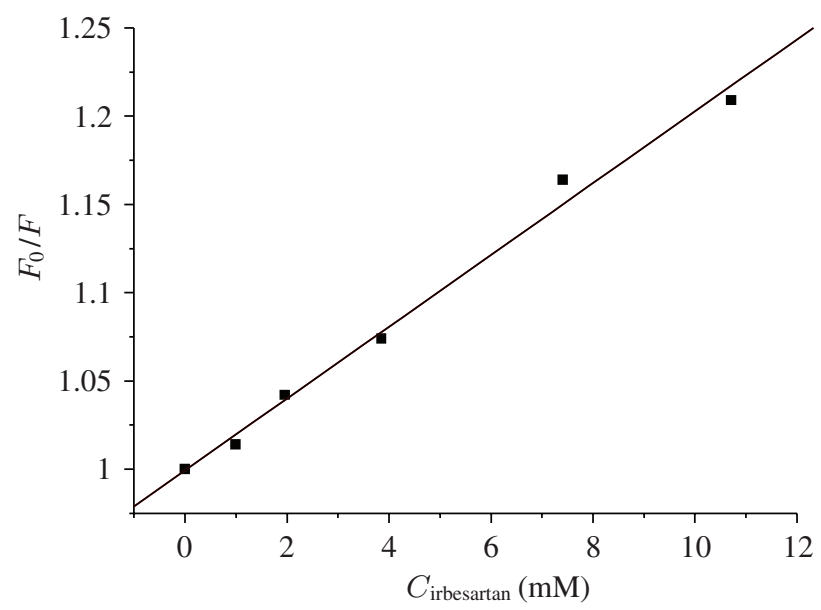

Figure 4: Stern-Volmer plots for the interaction of irbesartan and $\mathrm{BHb}$.

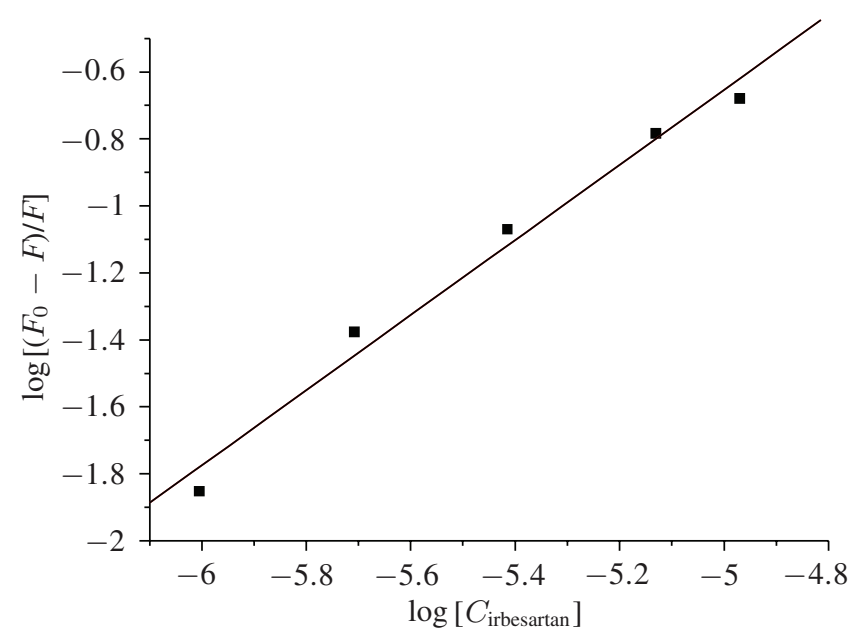

Figure 5: Plots of $\log \left[\left(F_{0}-F\right) / F\right]$ versus $\log \left[C_{\text {irbesartan }}\right]$.

using least-squares analysis whose slope was equal to $\mathrm{n}$ and the intercept on $y$-axis to $\log K$. The value of $n$ is approximately equal to 1 which indicated the existence of just a single binding site in $\mathrm{BHb}$ for irbesartan. The value of binding constant $K 2.41 \times 10^{5} \mathrm{M}^{-1}$ showed the strong interaction between $\mathrm{BHb}$ and irbesartan.

\subsection{Spectra Studies}

In order to get a better understanding in physicochemical properties of irbesartan governing its spectral behavior and to draw relevant conclusions on the irbesartan-BHb binding mechanism, CD spectroscopic measurements were performed on $\mathrm{BHb}$ and the irbesartan- $\mathrm{BHb}$ complex. If the change of protein structure included the transforming of protein secondary structure in the drug-protein complex, it can be 


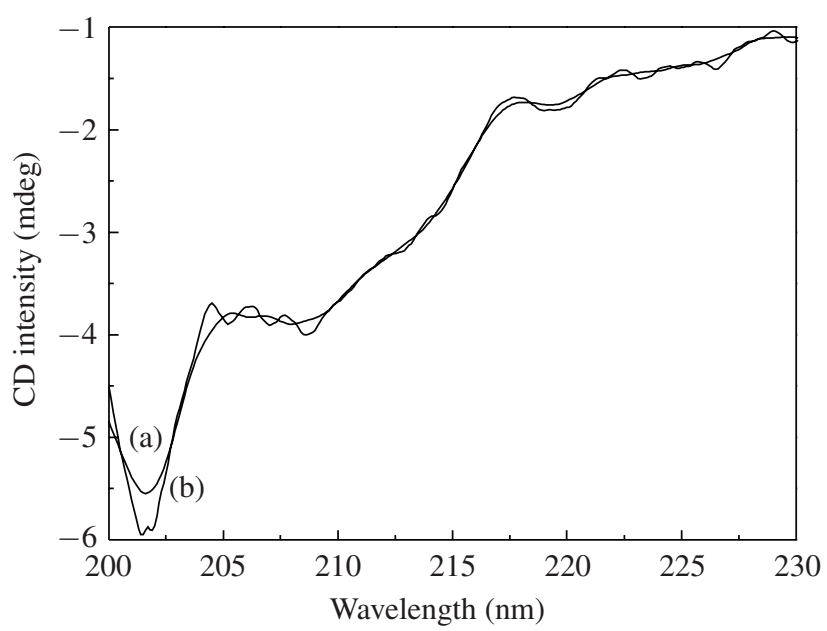

Figure 6: $\mathrm{CD}$ spectra of the BHb-irbesartan system. (a) $5 \times 10^{-6} \mathrm{M} \mathrm{BHb}$; (b) irbesartan: $\mathrm{BHb}=0.4$; $\mathrm{pH}$ 7.4.

reflected in the CD spectra. In Figure 6(a), the CD spectrum of free BHb in buffer solution exhibited an intensive negative band in the ultraviolet region at 208 and $219 \mathrm{~nm}$, which is a characteristic of $\alpha$-helical structure of protein [9]. As shown in Figure 6, the interaction between irbesartan and BHb caused only a decrease in negative ellipticity at all wavelength of the far-UV CD without any significant shift of the peaks, indicating that this drug induces a sight decrease in the helical structure content of protein. It is noteworthy that the $\mathrm{CD}$ spectra of $\mathrm{BHb}$ in the absence and presence of irbesartan are similar in shape, indicating that the structure of $\mathrm{BHb}$ is also predominantly $\alpha$-helical [10]. In conclusion, it is apparent that the affect of irbesartan on $\mathrm{BHb}$ caused a conformational change of the protein, with the loss of $\alpha$-helical stability. It is showed that the binding between irbesartan and $\mathrm{BHb}$ changed the secondary structure of $\mathrm{BHb}$, and it may be due to the formation of irbesartan-BHb complex.

\subsection{Molecule Docking}

There are four chains in the $\mathrm{BHb}$ molecule, named A, B, C, and D. For the docking experiment, the cavities in the $\mathrm{BHb}$ were defined as the active sites of $\mathrm{BHb}$. The number of 15 active sites has been found in the $\mathrm{BHb}$ molecule according to the docking procedure. To establish which binding site of $\mathrm{BHb}$ that irbesartan is located in, the complementary applications of molecule docking have been employed by computer methods to improve the understanding of the interaction of irbesartan and $\mathrm{BHb}$. The molecule docking was investigated through the flexible docking protocol in the DS 2.1 software.

Flexible docking means that the side chains can rotate continuously about single bonds during the docking simulation. The active-site atoms of the receptor were defined as those atoms within a radius of 9.0 $\AA$ from the ligand co crystallized with that particular receptor. Here, the conformational space of the ligand irbesartan is explored within the defined active-site of the $\mathrm{BHb}$. The nine ligand poses were generated by the flexible docking protocol, and all the irbesartan structures were scored by means of the libdock scoring function and scored in increasing orders of their scores. The higher the libdock score, the better the energy ranked [11]. Considering the CDOCK energy, CDOCK interaction energy and the 


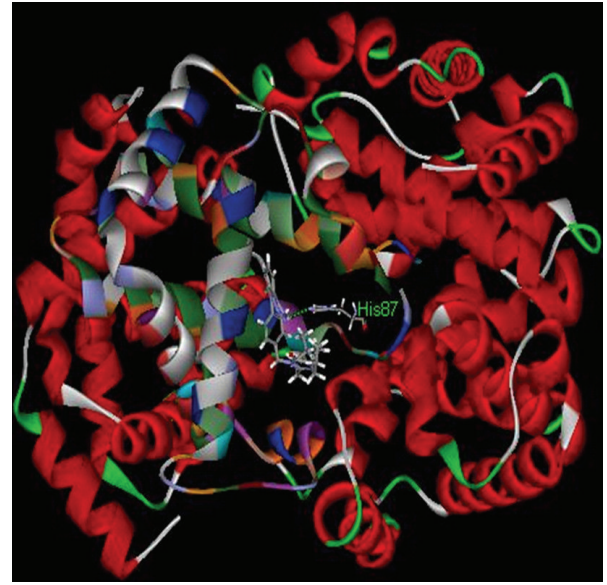

(a)

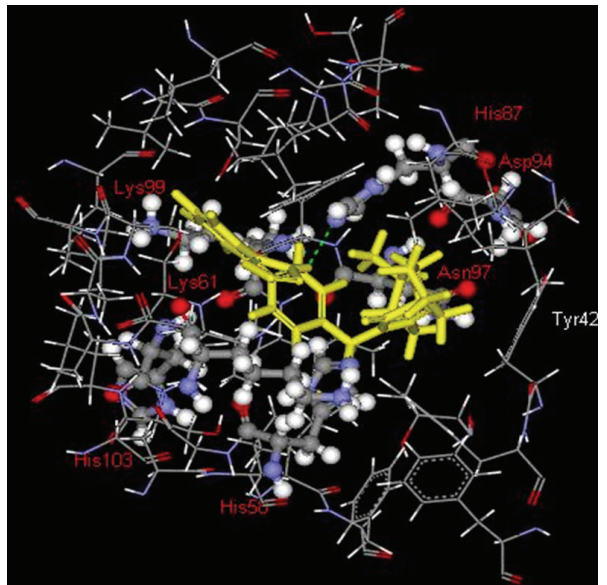

(b)

Figure 7: The interaction between irbesartan and $\mathrm{BHb}$, (a) an overview of the irbesartan molecular (stick molecular) located in $\mathrm{BHb}$; (b) the binding mode between irbesartan and the defined flexible residues in the $\mathrm{BHb}$.

number of hydrogen bond, two of the appropriate irbesartan poses were selected. In order to confirm which pose is the best energy-ranked result, the solvation, minimization, and calculation of binding free energy protocols were applied to calculate the protein-ligand complex. Generally speaking, the higher the binding free energy, the more suitable the ligand poses. According to the theoretical calculation, the best energy-ranked result was presented in Figure 7.

Figure 7(a) gave an overview of the irbesartan (stick molecule) located in BHb protein, from which it can be seen that irbesartan is located within the binding pocket of site 2, and site 1 is too large to accommodate the irbesartan molecule. As shown in Figure 7(a), the red color in the BHb molecule stands for the $\alpha$-helical, and most of the BHb protein is consisted by $\alpha$-helical. This is in good agreement with the CD spectrum results. Figure 7(b) showed the interaction details between the residues surround the defined sphere and irbesartan. It is important to note that the framework of the benzene ring in irbesartan is easy to interact with the hydrophobic amino acid residues, including Phe residue, which can produce UV-Vis absorption peak. It is the evidence that the existence of hydrophobic interaction between irbesartan and BHb leads to the decrease of the UV-Vis absorption peak of BHb. And the only Tyr residue (Tyr 42) of $\mathrm{BHb}$ is in close proximity the five-membered ring in the irbesartan, suggesting the existence of hydrophobic interaction between them. Further, this finding provides a good structural basis to explain the efficient fluorescence quenching of BHb emission in the presence of irbesartan.

In Figure 7(b), the hydrophilic amino acid residues were showen as ball and stick and labeled as red colour, the hydrophobic amino acid residues were showen as linear. However, the results obtained from molecule docking indicate that the interaction between irbesartan and BHb is not exclusively hydrophobic in nature since the several ionic and polar residues in the proximity of the ligand playing important role in stabilizing the drug molecule via hydrogen bond and electrostatic interaction. For instance, Histidine residue (His 87) is in suitable position to form intermolecular H-bond interaction with $17-\mathrm{N}$ in irbesartan. The length of hydrogen bond between them is $2.476 \AA$ and the angle of $\mathrm{H}$ bond is $122.3^{\circ}$. It is the evidence that the strong interaction is existed between two of them. This is 
in good agreement with the results of the UV/Vis absorption, fluorescence, and CD experiments. The results suggested that the formation of hydrogen bond decreased the hydrophilicity and increased the hydrophobicity to stability in the irbesartan-BHb system. It was obtained from the theoretical calculation that the values of electrostatic energy, van der Waals energy, and binding free energy of the interaction between irbesartan and $\mathrm{BHb}$ are $-460.3,-224.2$, and $-684.5 \mathrm{kcal} \cdot \mathrm{mol}^{-1}$, respectively.

\section{Conclusions}

In the present work, the binding of irbesartan and $\mathrm{BHb}$ under physiological conditions has been studied using molecular docking and several optical techniques. Experimental results showed that the binding between irbesartan and $\mathrm{BHb}$ induced a conformational change of $\mathrm{BHb}$, which was further proved by UV-Vis and CD spectrum. The results of fluorescence quenching measurements and molecular docking study suggested that irbesartan could bind to $\mathrm{BHb}$ through the hydrophobic interaction and hydrogen bond with high affinity. The binding site is located in the site 2 pocket of $\mathrm{BHb}$ according to the molecular docking study. The CD spectroscopic showed that the secondary structure of BHb changed after irbesartan bound to $\mathrm{BHb}$. The number of binding site and the binding constant between the drug and $\mathrm{BHb}$ was calculated based on the fluorescence experiment results. The binding study of irbesartan with $\mathrm{BHb}$ is of great importance in pharmacy, pharmacology, and biochemistry. This experiment can supply important information to clinical research and provide the theoretical basis for drug designing.

\section{References}

[1] X. Y. Bao, Z. W. Zhu, N. Q. Li, and J. G. Chen, "Electrochemical studies of rutin interacting with hemoglobin and determination of hemoglobin," Talanta, vol. 54, no. 4, pp. 591-596, 2001.

[2] C. Y. Chen, B. Zhao, and Z. W. Wang, "Interaction of thiacloprid with bovine hemoglobin using spectroscopic and molecular modeling methods," Spectroscopy, vol. 24, no. 5, pp. 559-566, 2010.

[3] C. Y. Chen, X. T. Gu, and J. H. Zhou, "Binding studies of paeonolum with bovine serum albumin using spectroscopic methods," Spectroscopy, vol. 21, no. 1, pp. 53-60, 2007.

[4] J. P. Voigt, B. Peter, and F. Heidrun, "Hypophagic effect of the angiotensin AT1 receptor antagonist irbesartan in rats," European Journal of Pharmacology, vol. 564, no. 1-3, pp. 131-137, 2007.

[5] C. Holger, B. Christian, R. Matthias, and T. Lengauer, "FLEXE: efficient molecular docking considering protein structure variations," Journal of Molecular Biology, vol. 308, no. 2, pp. 377395, 2001.

[6] Y. H. Zhang, L. J. Dong, J. Z. Li, and X. G. Chen, "Studies on the interaction of gallic acid with human serum albumin in membrane mimetic environments," Talanta, vol. 76, no. 2, pp. 246-253, 2008.

[7] C. Y. Chen, M. H. Ma, J. Q. Zhang, L. C. Wang, and B. R. Xiang, "Spectroscopic investigation of the interaction of bovine serum albumin with a novel cardiac agent V-09," Spectroscopy, vol. 22, no. 1, pp. 43-50, 2008.

[8] C. Y. Chen, K. Chen, Q. Long, M. H. Ma, and F. Ding, "Structural characterization and DNAbinding properties of Sm(III) complex with ofloxacin using spectroscopic methods," Spectroscopy, vol. 23, no. 2, pp. 103-111, 2009.

[9] S. Sugio, A. Kashima, S. Mochizuki, M. Noda, and K. Kobayashi, "Crystal structure of human serum albumin at 2.5 Å resolution,” Protein Engineering, vol. 12, no. 6, pp. 439-446, 1999. 
[10] H. R. Mohammed, M. Toru, O. Tomoko, I. Teruko, and M. Otagiri, "Study of interaction of carprofen and its enantiomers with human serum albumin-II. Stereoselective site-to-site displacement of carprofen by ibuprofen," Biochemical Pharmacology, vol. 46, no. 10, pp. 1733-1740, 1993.

[11] B. Giovanni, K. Irina, T. Maxim, and A. Ruben, "A new method for ligand docking to flexible receptors by dual alanine scanning and refinement (SCARE)," Journal of Computer-Aided Molecular Design, vol. 22, no. 5, pp. 311-325, 2008. 


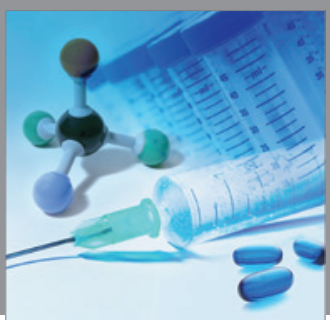

International Journal of

Medicinal Chemistry

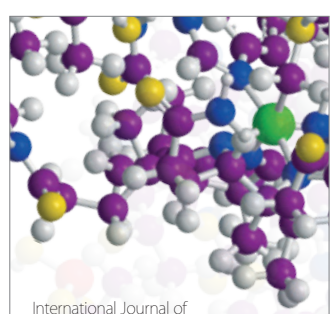

Carbohydrate Chemistry

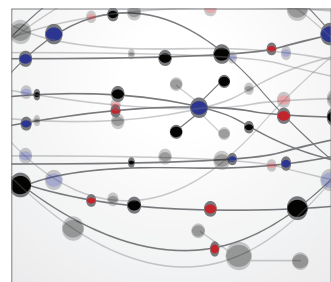

The Scientific World Journal
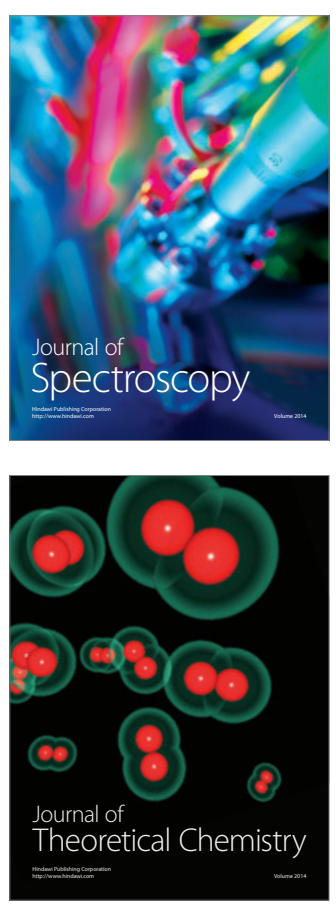
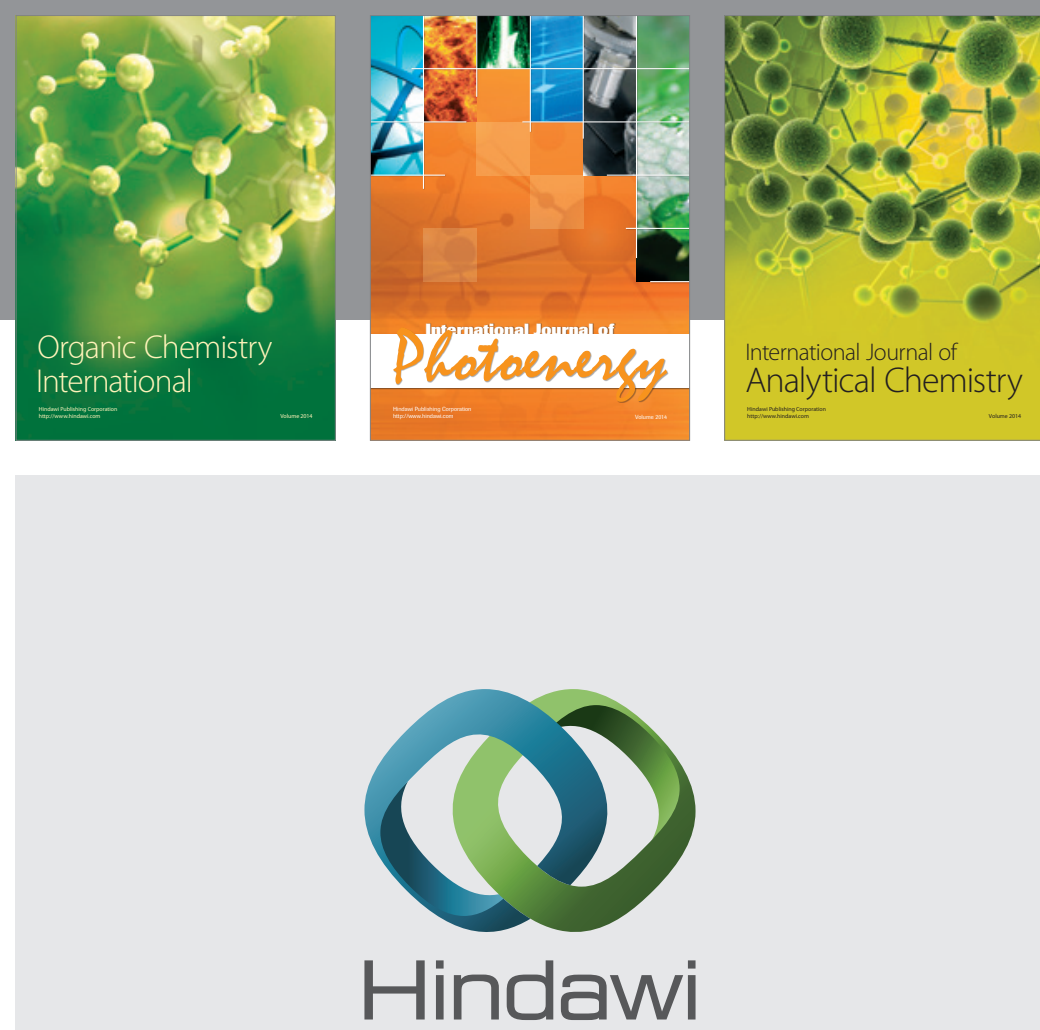

Submit your manuscripts at

http://www.hindawi.com
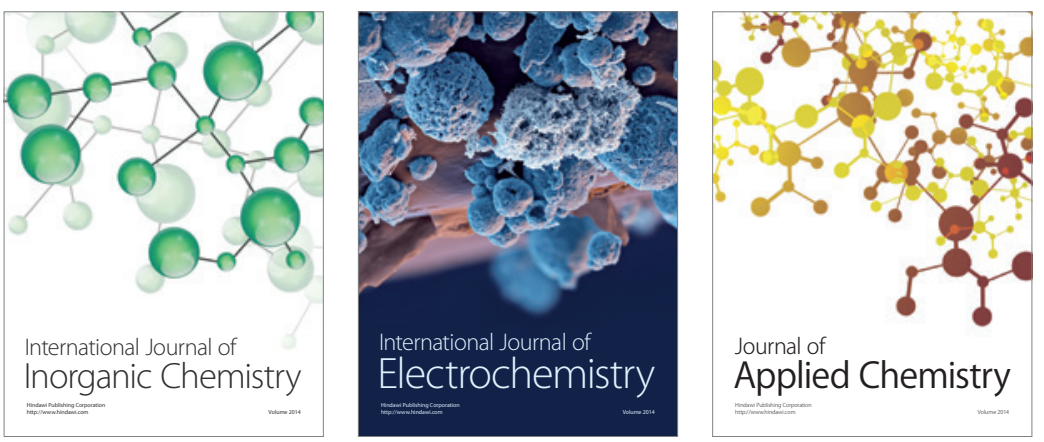

Journal of

Applied Chemistry
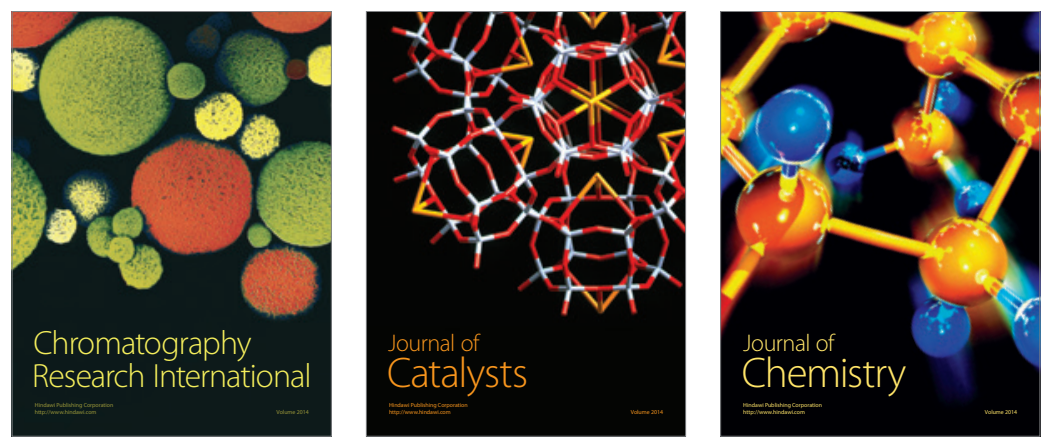
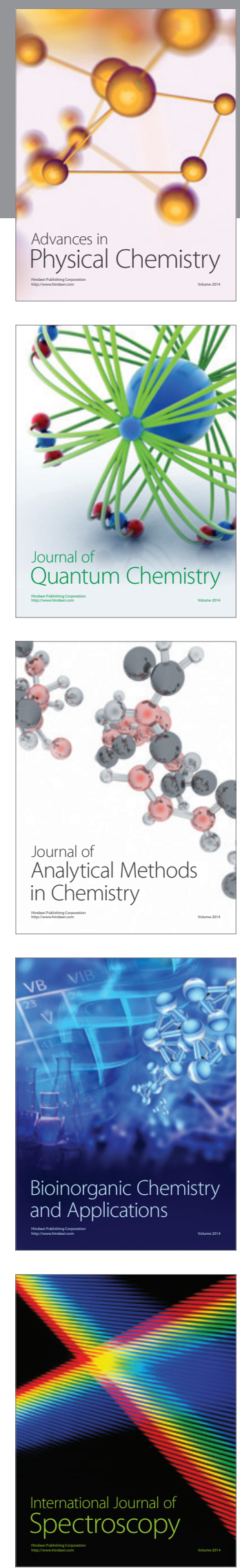